\title{
Fabrication of Microspheres Based on Poly(4-butyltriphenylamine) Blends with Poly(methyl methacrylate) and Block Copolymer by Solvent Evaporation Method
}

\author{
Hossein Taherzadeh, Kenji Ogino* \\ Graduate School of Bio-Applications and Systems Engineering, Tokyo University of Agriculture and Technology, \\ Koganei, Tokyo, Japan \\ Email: ${ }^{*}$ kogino@cc.tuat.ac.jp
}

Received 24 February 2015; accepted 17 March 2015; published 23 March 2015

Copyright (C) 2015 by authors and Scientific Research Publishing Inc. This work is licensed under the Creative Commons Attribution International License (CC BY). http://creativecommons.org/licenses/by/4.0/

\begin{abstract}
Micron-sized polymer particles from single poly(4-butyltriphenylamine) (PBTPA) homopolymer, binary polymer blend [PBTPA/poly(methyl methacrylate) (PMMA)], and ternary polymer blend (PBTPA/PBTPA-b-PMMA/PMMA) via a solvent evaporation method, and the surface morphologies and inside structure of resulting particles were investigated. Spherical homopolymer particles with smooth surface were resulted from PBTPA with low molecular weight. In the case of binary blends (PBTPA/PMMA = 1/1), Janus (low molecular weight) and dumbbell (high molecular weight) type morphologies were observed. The particles based on ternary blends containing PBTPA- $b$-PMMA showed core-shell type morphologies (PMMA; core, PBTPA; shell). Degree of engulfment of PMMArich domain increased with the content of the block copolymer. The decrease of domain size was not observed although the block copolymer had a suitable structure as a compatibilizer for the blend. It was also found that the initial concentration of polymer solution had an effect on the final morphology.
\end{abstract}

\section{Keywords}

Microsphere, Morphology, Polymer Blend, Block Copolymer, Poly(4-butyltriphenylamine)

\footnotetext{
${ }^{*}$ Corresponding author.
} 


\section{Introduction}

Triarylamine derivatives have been used as hole-transporting materials in organic photoconductors and electroluminescent devices [1] [2]. Our group originally synthesized polyarylamines (PAAs) where triarylamine units were linked together at para positions by chemical oxidative polymerization using ferric chloride as an oxidant [3]. Mono-butyl substituted PAA and poly(4-butyltriphenylamine) (PBTPA) showed the excellent photoconductivity compared with a conventional poly (N-vinylcarbazole) [4]. Palladium catalyzed C-N coupling reaction is also applied to prepare PAA and block copolymers consisting of PAA block from A-B type monomer [5] [6]. PAAs are also promising materials for photovoltaic [7], and photorefractive [8] [9] applications.

Meanwhile, polymeric microspheres have attracted much interest and have been utilized in a number of fields. As for PAAs, we reported PBTPA microspheres [10] and PBTPA/polystyrene (PSt) composite microspheres [11] prepared by chemical oxidative dispersion polymerization and seeded polymerization of BTPA, respectively.

Morphologically, controlled microspheres have been widely investigated. For conventional vinyl polymers, seeded emulsion [12]-[14], dispersion [15]-[17], and suspension [18] polymerizations have been utilized to obtain particles with the unique morphologies. In order to fabricate nanoparticles with inner micro-phase separated structure, Yabu et al. reported self-organized precipitation method [19] [20] in which a good solvent was evaporated from a block copolymer solution containing poor and good solvents. In order to fabricate the microspheres from polymer solutions, the other type of solvent evaporation method has been used, where a polymer solution is dispersed in an aqueous medium, and then the solvent is evaporated to solidify the polymer [21]-[23]. Phase-separation during the solvent evaporation afforded core-shell, and inverted core-shell, microdomain, hemisphere morphologies [21] and non-spherical shape of polystyrene (PSt)/poly(methyl methacrylate) (PMMA) composite [22] [23].

In this study, the solvent evaporation method was applied to fabricate the microspheres based on PBTPA/ PMMA binary blends, and PBTPA/PBTPA- $b$-PMMA/PMMA ternary blends, and the relationship between the fabrication conditions and the morphologies was investigated. It is found that molecular weight of PBTPA and the concentration of original polymer solutions have a significant effect on the morphologies of the resulting particles.

\section{Experimental}

\subsection{Materials}

Two PBTPA homopolymers with different molecular weight (PBTPA-L; $M_{\mathrm{n}}=3700$, and PBTPA-H; $M_{\mathrm{n}}=6400$ ) were synthesized via palladium-catalyzed $\mathrm{C}-\mathrm{N}$ coupling polymerization [5]. The number average molecular weight $\left(M_{\mathrm{n}}\right)$ was determined by an end-group analysis with ${ }^{1} \mathrm{H}-\mathrm{NMR}$. Poly(methyl methacrylate) (PMMA) was prepared by an anionic polymerization as described elsewhere [24]. The block copolymer PBTPA- $b$-PMMA with the number average molecular weight of 8500 (PBTPA; $M_{\mathrm{n}}=4300$, PMMA; $M_{\mathrm{n}}=4200$ ) was synthesized by the combination of $\mathrm{C}-\mathrm{N}$ coupling polymerization and atom transfer radical polymerization (ATRP) of MMA as follows. At first bromo ester terminated macroinitiotor PBTPA-MI $\left(M_{\mathrm{n}}=4300\right)$ was prepared as described elsewhere [7]. Then ATRP of MMA was carried out.

A 5-mL flask equipped with a magnetic stirrer and a nitrogen inlet was charged with PBTPA-MI $(0.13 \mathrm{~g}, 0.03$ $\mathrm{mmol})$ and $\mathrm{CuBr}(94 \mathrm{mg}, 0.66 \mathrm{mmol})$. After the evacuation followed with backfilling of nitrogen, anisole $(1 \mathrm{~mL})$, distilled MMA (0.63 mL, $6.0 \mathrm{mmol})$ and N,N,N',N",N"-pentamethyldiethylenetriamine (PMDETA) $(127 \mu \mathrm{L}$, $0.62 \mathrm{mmol}$ ) were added. Then the reaction mixture was stirred for $5 \mathrm{~min}$. After five freeze-pump-thaw cycles, the flask was placed in a $100^{\circ} \mathrm{C}$ oil bath for $24 \mathrm{~h}$. After dilution with THF, the reaction mixture was filtered with alumina column in order to remove the copper catalyst. After concentration, the polymer was obtained by precipitation from methanol $(1.70 \mathrm{~g})$. Judging from GPC trace and ${ }^{1} \mathrm{H}-\mathrm{NMR}$ spectrum, no homopolymer was contained in the product.

Chloroform (analytical grade) was used as a volatile solvent for a solvent evaporation method. Poly (vinyl alcohol) (PVA) (Kuraray, PVA224) as a stabilizer was used as received.

\subsection{Preparation of Microsphere}

A polymer solution (in chloroform, $2.5 \%$ or $5 \%$ ) was dispersed in ten times volume of water containing $0.3 \%$ of PVA with a homogenizer (X520, CAT Scientific, US) to obtain $2-10 \mu \mathrm{m}$ sized droplets (usually 5 min treat- 
ment).The weight ratio for the binary blend was fixed to be 1/1 (PBTPA/PMMA). In the case of ternary blends, the content of block copolymer was 10,30 or $50 \mathrm{wt} \%$ keeping the weight ratio of PBTPA component to PMMA component constant (1/1). The homogenized mixture was stirred by a mechanical stirrer at $100 \mathrm{rpm}$ for $24 \mathrm{hr}$ to evaporated $\mathrm{CHCl}_{3}$ slowly. The resulting microspheres were washed with distilled water four times with a centrifugation process. Finally, polymer seeds dried under vacuum.

\subsection{Characterization}

Resulting PBTPA and PBTPA- $b$-PMMA were characterized with ${ }^{1}$ H-NMR (ECX 300, JEOL, Japan) and GPC [18]. The surface feature and inside morphologies of microspheres were characterized by scanning electron microscope (SEM) (JSM-6510, JEOL, Japan) and transmission electron microscope (TEM) (JEM-2100, JEOL, Japan), respectively. The specimens for SEM and TEM observations were prepared as described elsewhere [24]. The PBTPA domain was stained by exposing the specimens to the vapor of an aqueous $\mathrm{RuO}_{4}$ solution $(0.5 \%)$ for $90 \mathrm{~min}$ in a sealed bottle at room temperature.

\section{Results and Discussion}

\subsection{Particles Based on PBTPA Homopolymer and Binary Blends with PMMA}

Figure 1 shows SEM images of microspheres with PBTPA homopolymer fabricated at the initial polymer concentration of 5\%. As shown in Figure 1(a), low molecular weight polymer (PBTPA-L) afforded spherical polymer particles with external smooth surface. In the case of high molecular weight polymer (PBTPA-H), Irregular and in some extents, non-spherical larger particles with rough surfaces were produced even at the same experimental conditions (Figure 1(b)). Since the both polymers exhibit the same solubility (miscible in chloroform), it is considered that slightly increased viscosity of the solution made resulting particle irregular and larger in the case of PBTPA-H.

In next step, microspheres based on the binary blend PBTPA/PMMA (weight ratio 1/1) were fabricated, and the effect of molecular weight of PBTPA was investigated. Figure 2 shows microscopic images for the resulting particles $(\mathrm{a}, \mathrm{c})$ and dispersed polymer solutions (b, d). Basically bi-compartmental morphologies were observed for both composites. In the case of PSt/PMMA blend (weight ratio 1/1) particles, core-shell morphology is observed when a PVA solution is used as a continuous phase [21] [24].

As Ge et al. discussed thermodynamically [25], the interfacial tension between dichloromethane solution of PSt and water at the point of the phase separation should be larger compared with the sum of that between two polymer solutions and that between PMMA solution and water for the core-shell morphology (PMMA; shell, and PSt; core). In this study, it is reasonable that the interfacial tension between chloroform solution of PBTPA and water decreased compared with PSt due to the presence of nitrogen atoms, and is comparable to that between chloroform solution of PMMA and water. PBTPA-L/PMMA composites afforded a Janus like morphology, whereas PBTPA-H/PMMA a dumbbell-like morphology. This difference is probably due to the molecular weight dependence of the interfacial tension between both polymer solutions [25] [26]. Figure 2(b) and Figure 2(d) show the optical micrographs for the dispersions just after the dispersing processes. Those images indicate that phase separation of both PBTPA and PMMA solutions occurs at an early stage in the solvent evaporation process, and the morphology at phase separation retains in final particles. It is noteworthy that the binary blend based on PBTPA-H provided particles with a regular shape and surface smooth morphology compared with PBTPA-H homopolymer.

\subsection{Particles Based on PBTPA/PBTPA-b-PMMA/PMMA Ternary Blends}

In order to investigate the effect of block copolymer on the surface and inside morphologies, various amount of PBTPA- $b$-PMMA was added to the binary blend. All ternary blend particles were fabricated keeping the weight ratio of PBTPA component to PMMA component constant (1/1), and low molecular weight PBTPA-L was utilized. Figure 3 shows SEM images for the particles based on ternary blends. The effect of the ratio of the block copolymer [(a), (b): 10\%; (c), (d): 30\%; (e), (f); 50\%], and the initial concentration dependence on the morphology [(a), (c), (d): 5\%; (b), (d), (f): 2.5\%] were also investigated. By adding of block copolymer, bi-compartmental microspheres observed in the binary blends particles were not formed. Ternary blends with $10 \%$ of the block copolymers at the initial concentration of $5 \%$ afforded particles with some patches on the surface. In 
the high concentration regions, it is possible that microdomain type, a nonequilibrium morphology is observed, because of high viscosity in the droplets [21]. Particles were fabricated at low initial concentration to assure the equilibrium in the formation of particles. However, as shown in Figure 3(b), more irregular shape was observed at $2.5 \%$. At $5 \%$ of total polymer concentration, the increase of the ratio of the block copolymer made the surface smoother as shown in Figure 3(c) and Figure 3(e). At 30\%, particles seem to have navel. At 50\%, smooth and spherical particles were obtained. At both block copolymer contents, the low initial concentration afforded particles with irregular shapes as shown in Figure 3(d) and Figure 3(f).

In order to investigate morphologies inside microspheres and to explore the origin of unique surface features, TEM measurements were carried out. Figure 4 shows TEM images of microspheres based on ternary blends fabricated from $5 \%$ of polymer solutions. At $10 \%$ of the block copolymer content, the Janus type morphology was no longer observed, and PMMA-rich bright domain was partially engulfed by PBTPA-rich dark domain as shown in Figure 4(a). It is considered that protruded PMMA rich domain formed patch like morphologies at the surface. At 30\%, PMMA-rich domain was almost completely engulfed as shown in Figure 4(b). The formation of navels is attributed to the contact point of two domains near the surface. At $50 \%$, a nearly concentric circle image (Figure 4(c)) exhibits the formation of particles with core-shell morphology. It is generally accepted that the block copolymer plays a role of a compatibilizer and the addition of block copolymer decrease the difference of the interfacial tension between two polymer solutions and solids [27]. Therefore it is expected that size of phase separated domain is decreased. In this experiment, however, the formation of microdomains was not observed, and the type of morphology changed by the addition of block copolymers. In our previous study for the blend of PSt and PMMA, the morphologies changes from core-shell to Janus by the addition of the corresponding block copolymers. Until now it is difficult to explain the discrepancy, and the further study is necessary.
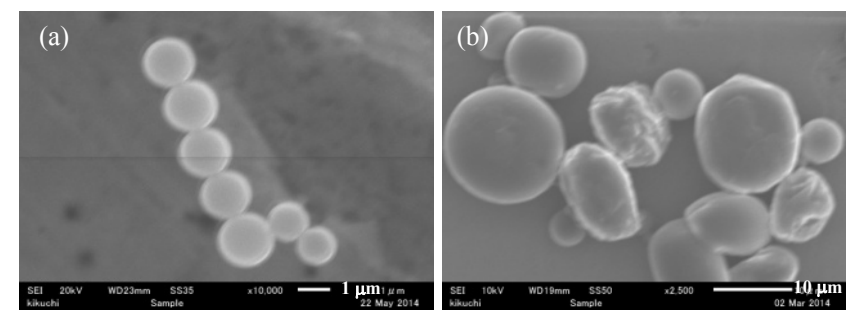

Figure 1. SEM images of particles based on PBTPA homopolymers with low (a) and high (b) molecular weight.
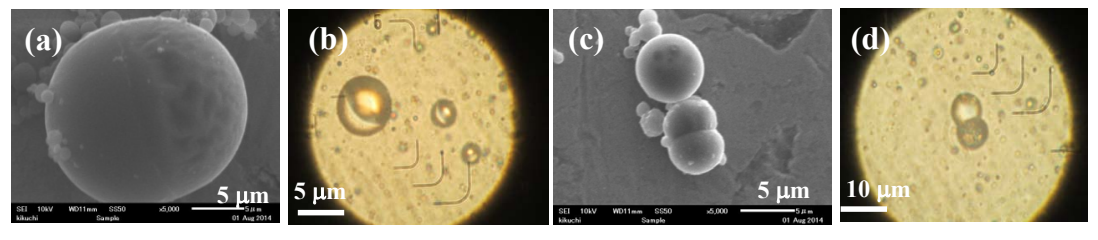

Figure 2. Micrographs of binary blends consisting of PBTPA and PMMA. SEM images of polymer particles with (a) PBTPA-L; (c) PBTPA-H, optical microscope images of solution droplets with; (b) PBTPA-L; (d) PBTPA-H.
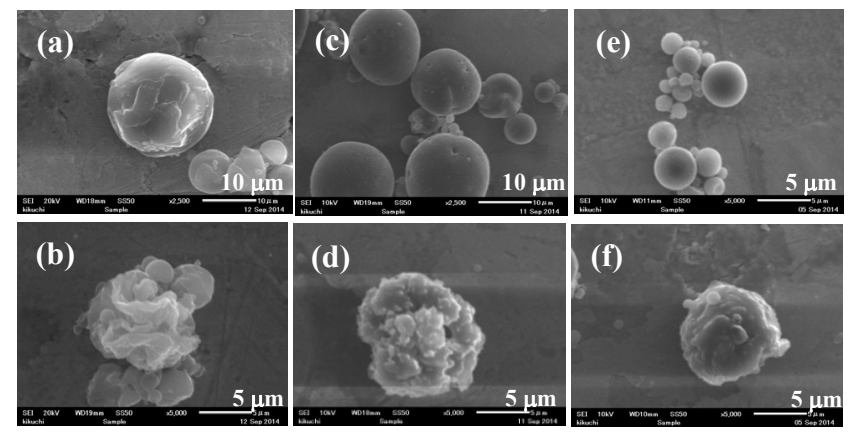

Figure 3. SEM images of microspheres based on PBTPA/PBTPA- $b$-PMMA/PMMA ternary blends. The content of PBTPA- $b$-PMMA is (a) (b) $10 \mathrm{wt} \%$; (c) (d) $30 \mathrm{wt} \%$; (e) (f) $50 \mathrm{wt} \%$. The initial concentration of polymer solutions is (a) (c) (e) $5 \%$; (b) (d) (f) $2.5 \%$. 

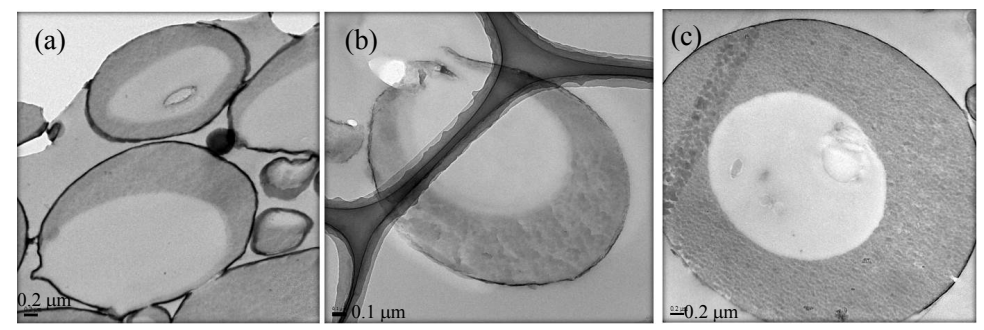

Figure 4. TEM images of microspheres based on PBTPA/PBTPA- $b$-PMMA/PMMA ternary blends. The content of PBTPA- $b$-PMMA is (a) $10 \mathrm{wt} \%$; (b) $30 \mathrm{wt} \%$; (c) $50 \mathrm{wt} \%$.

\section{Conclusion}

PBTPA homopolymer with low molecular weight (PBTPA-L) afforded spherical particles on the conditions we examined in a solvent evaporation method. On the other hand, the particles based on PBTPA with high molecular weight (PBTPA-H) were non-spherical and irregular. Unique bi-compartmental particles were obtained from binary blends with PMMA. Molecular weight dependence on the final morphologies was also observed, i.e. the particles based on PBTPA-L/PMMA and PBTPA-H/PMMA exhibited Janus and dumbbell type morphologies, respectively. This observation is reasonably explained by molecular weight dependence of the interfacial tension between two kinds of phase separated polymer solutions. Bi-compartmental morphologies were no longer observed for the particles based on ternary blends containing PBTPA- $b$-PMMA. Instead, the particles showed core-shell (PMMA; core, PBTPA; shell) morphologies. As the content of the block copolymer increased, the extent of engulfment of PMMA domain increased. Since physical properties such as dielectric constant and refractive index of PBTPA are considerably different from those of PMMA, the particles with various morphologies have potential applications in photonic and electronic fields.

\section{References}

[1] Adachi, C. and Tsutsui, T. (1999) Molecular LED: Design Concept of Molecular Materials for High Performance OLED. In: Miyata, S. and Nalwa, H.S., Ed., Organic Electroluminescence Materials and Device, Gordon and Breach, Amsterdam, 43-65.

[2] Tang, C.W. and VanSlyke, S.A. (1987) Organic Electroluminescent Diodes. Applied Physics Letters, 51, $913-915$. http://dx.doi.org/10.1063/1.98799

[3] Ogino, K., Kanegae, A., Yamaguchi, R., Sato, H. and Kurjata, J. (1999) Oxidative Coupling Polymerization of 4-Methyltriphenylamine. Macromolecular Rapid Communications, 20, 103-106. http://dx.doi.org/10.1002/(SICI)1521-3927(19990301)20:3<103::AID-MARC103>3.0.CO;2-Q

[4] Takahashi, C., Moriya, S., Fugono, N., Lee, H.C. and Sato, H. (2002) Preparation and Characterization of Poly(4-alkyltriphenylamine) by Chemical Oxidative Polymerization. Synthetic Metals, 129, 123-128. http://dx.doi.org/10.1016/S0379-6779(02)00010-3

[5] Tsuchiya, K., Shimomura, T. and Ogino, K. (2009) Preparation of Diblock Copolymer Based on Poly(4- $n$-butyltriphenylamine) via Palladium Coupling Polymerization. Polymer, 50, 95-101. http://dx.doi.org/10.1016/j.polymer.2008.10.057

[6] Tan, Y., Gu, Z., Tsuchiya, K. and Ogino, K. (2012) Synthesis and Luminescent Properties of Block Copolymers Based on Polyfluorene and Polytriphenylamine. Polymer, 53, 1444-1452. http://dx.doi.org/10.1016/j.polymer.2012.02.021

[7] Tsuchiya, K., Kikuchi, T. Songeun, M., Shimomura, T. and Ogino, K. (2011) Synthesis of Diblock Copolymer Consisting of Poly(4-butyltriphenylamine) and Morphological Control in Photovoltaic Application. Polymers, 3, 10511064. http://dx.doi.org/10.3390/polym3031051

[8] Cao, Z., Abe, Y., Nagahama, T., Tsuchiya, K. and Ogino, K. (2013) Synthesis and Characterization of Polytriphenylamine Based Graft Polymers for Photorefractive Application. Polymer, 54, 269-276. http://dx.doi.org/10.1016/i.polymer.2012.11.028

[9] Kinashi, K., Shinkai, H., Sakai, W. and Tsutsumi, N. (2013) Photorefractive Device Using Self-Assembled Monolayer Coated Indium-Tin-Oxide Electrodes. Organic Electronics, 14, 2987-2993. http://dx.doi.org/10.1016/j.orgel.2013.08.024

[10] Fujioka, M., Kurihara, H., Kawamura, R., Sato, H., Tsuchiya, K. and Ogino, K. (2008) Preparation of Poly(4-butyltriphenylamine) Particles by Chemical Oxidative Dispersion Polymerization. Colloid and Polymer Science, 286, 313- 


\section{8. http://dx.doi.org/10.1007/s00396-007-1779-5}

[11] Fujioka, M., Sato, H. and Ogino, K. (2007) Preparation of Polystyrene/Poly(4-butyltriphenylamine) Composite Particles by Chemical Oxidative Seeded Dispersion Polymerization. Colloid and Polymer Science, 285, 915-921. http://dx.doi.org/10.1007/s00396-007-1639-3

[12] Zhao, J., Yuan, H. and Pan, Z. (1994) Grafting Mechanism in SBR-St-MMA Core-Shell Emulsion Polymerization. Journal of Applied Polymer Science, 53, 1447-1452. http://dx.doi.org/10.1002/app.1994.070531106

[13] Han, S.H., Ma, G.H., Du, Y.Z., Omi, S. and Gu, L.X. (2003) Preparation of Hemispherical Poly(4-vinylpyridine-co-butyl acrylate)/Poly(styrene-co-butyl acrylate) Composite Microspheres by Seeded Pre-Swelling Emulsion Polymerization. Journal of Applied Polymer Science, 90, 3811-3821. http://dx.doi.org/10.1002/app.13094

[14] Hu, G., Yu, D., Zhang, J., Liang, H. and Cao, Z. (2011) Synthesis of Micron-Sized Poly(styrene-co-divinylbenzene) Hollow Particles from Seeded Emulsion by Using Swelling Solvents. Colloid Journal, 73, 557-564. http://dx.doi.org/10.1134/S1061933X11040077

[15] Okubo, M., Fujibayashi, T., Yamada, M. and Minami, H. (2005) Micron-Sized, Monodisperse, Snowman/ConfettiShaped Polymer Particles by Seeded Dispersion Polymerization. Colloid and Polymer Science, 283, 1041-1045. http://dx.doi.org/10.1007/s00396-004-1240-y

[16] Fujibayashi, T., Tanaka, T., Minami, H. and Okubo, M. (2010) Thermodynamic and Kinetic Consideration on the Morphological Stability of Hamburger-Like Composite Polymer Particles Prepared by Seeded Dispersion Polymerization. Colloid and Polymer Science, 288, 879-886. http://dx.doi.org/10.1007/s00396-010-2211-0

[17] Okubo, M., Fujibayashi, T. and Terada, A. (2005) Synthesis of Micron-Sized, Monodisperse Polymer Particles of Disk-Like and Polyhedral Shapes by Seeded Dispersion Polymerization. Colloid and Polymer Science, 283, 793-798. http://dx.doi.org/10.1007/s00396-004-1210-4

[18] Ogino, K., Sato, H., Tsuchiya, K., Suzuki, H. and Moriguchi, S. (1995) Synthesis of Monodisperse Macroreticular Styrene-Divinylbenzene Gel Particles by a Single-Step Swelling and Polymerization Method. Journal of Chromatography A, 699, 59-69. http://dx.doi.org/10.1016/0021-9673(95)00031-H

[19] Higuchi, T., Tajima, A., Motoyoshi, K., Yabu, H. and Shimomura, M. (2009) Suprapolymer Structures from Nanostructured Polymer Particles. Angewandte Chemie International Edition, 48, 5125-5128. http://dx.doi.org/10.1002/anie.200900002

[20] Higuchi, T., Tajima, A., Yabu, H. and Shimomura, M. (2008) Spontaneous Formation of Polymer Nanoparticles with Inner Micro-Phase Separation Structures. Soft Matter, 4, 1302-1305. http://dx.doi.org/10.1039/b800904j

[21] Ma, G.H., Nagai, M. and Omi, S. (1999) Study on Preparation and Morphology of Uniform Artificial PolystyrenePoly(methyl methacrylate) Composite Microspheres by Employing the SPG Membrane Emulsification Technique. Journal of Colloid and Interface Science, 214, 264-282. http://dx.doi.org/10.1006/jcis.1999.6188

[22] Okubo, M., Saito, N. and Fujibayashi, T. (2005) Preparation of Polystyrene/Poly(methyl methacrylate) Composite Particles Having a Dent. Colloid and Polymer Science, 283, 691-698. http://dx.doi.org/10.1007/s00396-004-1209-X

[23] Saito, N., Kagari, Y. and Okubo, M. (2006) Effect of Colloidal Stabilizer on the Shape of Polystyrene Poly(methyl methacrylate) Composite Particles Prepared in Aqueous Medium by the Solvent Evaporation Method. Langmuir, 22, 9397-9402. http://dx.doi.org/10.1021/la061298v

[24] Hossein, T., Sotowa, S. and Ogino, K. (2015) Morphology Control of Polymer Microspheres Containing Block Copolymers with Seed Polymerization. Open Journal of Organic Polymer Materials, in Press.

[25] Ge, X., Wang, M., Ji, X., Ge, X. and Liu, H. (2009) Effects of Concentration of Nonionic Surfactant and Molecular Weight of Polymers on the Morphology of Anisotropic Polystyrene/Poly(methyl methacrylate) Composite Particles Prepared by Solvent Evaporation Method. Colloid and Polymer Science, 287, 819-827. http://dx.doi.org/10.1007/s00396-009-2036-X

[26] Tanaka, T., Nakatsuru, R., Kagari, Y., Saito, N. and Okubo, M. (2008) Effect of Molecular Weight on the Morphology of Polystyrene/Poly(methyl methacrylate) Composite Particles by the Solvent Evaporation Method. Langmuir, 24, 12267-12271. http://dx.doi.org/10.1021/la802287s

[27] Maric, M. and Macosko, C.W. (2002) Block Copolymer Compatibilizers for Polystyrene/Poly(dimethylsiloxane) Blends. Journal of Polymer Science Part B: Polymer Physics, 40, 346-357. http://dx.doi.org/10.1002/polb.10098 